Surgery for

Congenital Heart

Disease

\title{
Diagnostic power of aortic elastic properties in young patients with Marfan syndrome
}

Daniela Baumgartner, $\mathrm{MD},{ }^{\text {a }}$ Christian Baumgartner, $\mathrm{PhD},{ }^{\mathrm{b}}$

Gabor Mátyás, PhD, ${ }^{c, d}$ Beat Steinmann, $M D,{ }^{c}$ Judith Löffler-Ragg, MD, ${ }^{e}$

Elisabeth Schermer, $\mathrm{MD}^{\mathrm{a}}{ }^{\mathrm{a}}$ Ulrich Schweigmann, $\mathrm{MD},{ }^{\mathrm{a}}$ Ivo Baldissera, $\mathrm{MD}^{\mathrm{f}}$ Bernhard Frischhut, MD, ${ }^{\mathrm{g}}$ John Hess, MD, PhD, ${ }^{\mathrm{h}}$ and Ignaz Hammerer, $\mathrm{MD}^{\mathrm{a}}$

From the Department of Pediatric Cardiol-
ogy, Innsbruck Medical University, Inns-
bruck, Austria, ${ }^{\text {a }}$ Research Group for Bio-
medical Data Mining, Institute for
Information Systems, University for Health
Sciences, Medical Informatics and Tech-
nology, Innsbruck, Austria, ${ }^{\text {b }}$ Division of
Metabolism and Molecular Pediatrics, Uni-
versity Children's Hospital, Zurich, Swit-
zerland, ${ }^{\mathrm{c}}$ Institute of Medical Genetics,
University of Zurich, Schwerzenbach,
Switzerland, ${ }^{\mathrm{d}}$ Institute of Medical Biology
and Human Genetics, Innsbruck Medical
University, Innsbruck, Austria, ${ }^{\mathrm{e}}$ Depart-
ment of Ophthalmology, Innsbruck Medi-
cal University, Innsbruck, Austria, ${ }^{\mathrm{f}}$ Depart-
ment of Orthopedics, Innsbruck Medical
University, Innsbruck, Austria, ${ }^{\mathrm{g}}$ and De-
partment of Pediatric Cardiology and Con-
genital Heart Disease, German Heart Cen-
ter, Munich, Germany.

Supported by the Austrian Industrial Research Promotion Fund (grant HITT-10 UMIT), Wolfermann-Nägeli-Stiftung (Zurich, Switzerland), and the Swiss National Science Foundation (grant 3200-059 445/2).

Received for publication March 4, 2004; revisions received June 21, 2004; accepted for publication July 8, 2004.

Address for reprints: Daniela Baumgartner, MD, Department of Pediatric Cardiology, Innsbruck Medical University, Anichstr 35, A-6020 Innsbruck, Austria (E-mail: Daniela. Baumgartner@aon.at)

J Thorac Cardiovasc Surg 2005;129:730-9 $0022-5223 / \$ 30.00$

Copyright (C) 2005 by The American Association for Thoracic Surgery

doi:10.1016/j.jtcvs.2004.07.019
Background: In patients with Marfan syndrome, progressive aortic dilation implicates a still-unpredictable risk of life-threatening aortic dissection and rupture. We sought to quantify aortic wall dysfunction noninvasively, determine the diagnostic power of various aortic parameters, and establish a diagnostic model for the early detection of aortic abnormalities associated with Marfan syndrome.

Methods: In 19 patients with Marfan syndrome (age, $17.7 \pm 9.5$ years) and 19 ageand sex-matched healthy control subjects, computerized ascending and abdominal aortic wall contour analysis with continuous determination of aortic diameters was performed out of transthoracic M-mode echocardiographic tracings. After simultaneous oscillometric blood pressure measurement, aortic elastic properties were determined automatically.

Results: The following ascending aortic elastic parameters showed statistically significant differences between the Marfan group and the control group: (1) decreased aortic distensibility $(P<.001)$, (2) increased wall stiffness index $(P<.01)$, (3) decreased systolic diameter increase $(P<.01)$, and $(4)$ decreased maximum systolic area increase $(P<.001)$. The diagnostic power of all investigated parameters was tested by single logistic regression models. A multiple logistic regression model including solely aortic parameters yielded a sensitivity of $95 \%$ and a specificity of $100 \%$.

Conclusions: In young patients with Marfan syndrome, a computerized image-analyzing technique revealed decreased aortic elastic properties expressed by parameters showing high diagnostic power. A multiple logistic regression model including merely aortic parameters can serve as useful predictor for Marfan syndrome.

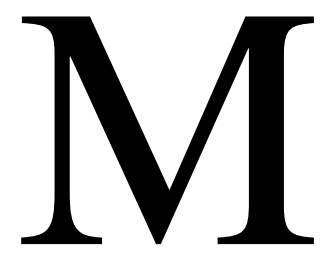

arfan syndrome (MFS; Online Mendelian Inheritance in Man $\# 154700)$ is an autosomal dominant connective tissue disorder caused by mutations in the gene encoding fibrillin-1 (FBN1), with highly variable clinical manifestations in the musculoskeletal, ocular, and cardiovascular systems. ${ }^{1,2}$ Dilatation of the aortic root predisposes the subject to aortic dissection and rupture or severe regurgitation and heart failure. ${ }^{3}$ Diseases of the 
aorta account for $80 \%$ of known causes of death. ${ }^{3}$ Before life-threatening complications, alterations of aortic elastic properties due to defective FBN1 can be characterized by the terms of elasticity or compliance, distensibility, stiffness index, and pulse wave velocity. ${ }^{4-7}$

The aim of this study was to investigate aortic elasticity and assess its abnormality in patients with MFS by means of a standardized, semiautomated, and noninvasive method. This technique is appropriate for determining the course of aortic elasticity during follow-up investigations. All aortic parameters were implemented in single logistic regression models to test their diagnostic power. To further increase sensitivity and specificity, we searched for a multiple logistic regression model able to serve as an appropriate diagnostic marker for MFS. To localize aortic elastic dysfunction, we suggest visualization of ascending (AscAo) and descending aortic (DescAo) diameter changes by a vector loop.

\section{Methods}

\section{Patients and Control Subjects}

Forty-seven people with suspected MFS were investigated at the Departments of Pediatric Cardiology, Ophthalmology, and Orthopedics and at the Institute of Medical Biology and Human Genetics, Innsbruck Medical University, according to a standardized protocol. Nineteen of these, whose diseases were diagnosed as MFS according to the Ghent criteria ${ }^{8}$ and who were younger than 40 years, comprised the study group ( 3 males and 16 females; mean age, $17.7 \pm 9.5$ years). Clinical characteristics are shown in Tables 1 and 2. Physical features were documented according to the consensus of 2 physicians (D.B. and J.L.-R.). Before the investigation, no patient received a $\beta$-blocker, angiotensin-converting enzyme inhibitor, or calcium antagonist or had a history of aortic dissection or aortic surgery. Nineteen age- and sex-matched healthy subjects constituted the control group. Two of them were healthy relatives of patients with MFS. A group of 35 people totally different from the study population, including 16 patients with MFS and 19 healthy controls, served as validation group for the logistic regression analysis. The mean age of this group was $14.2 \pm 8.0$ years and ranged from 0 to 36 years. The study complied with the Declaration of Helsinki. The protocol was approved by the institutional committee on human research. All subjects gave informed consent.

\section{Molecular Genetic Analysis}

Mutation analysis of the FBN1 gene was performed in all 19 MFS patients as described. ${ }^{9}$ In brief, genomic DNA samples were amplified exon by exon by means of polymerase chain reaction (PCR) by using intron-specific primers. All 65 amplicons were analyzed by denaturing high-performance liquid chromatography followed by direct sequencing of amplicons with abnormal elution profiles. The mutations found were verified by repeated sequencing on newly amplified PCR products. In the case of splice site mutations and when no mutation was detected by denaturing high-performance liquid chromatography, FBN1 transcripts were analyzed by reverse transcription-PCR of RNA templates isolated from fibroblasts.

\section{Echocardiographic Evaluation}

All echocardiographic examinations were performed by 1 investigator (D.B.) in the left decubitus position with commercially available equipment (System Five; GE Vingmed Ultrasound, Horten, Norway). M-mode tracings of the aorta were obtained according to published criteria ${ }^{10}$ by using 2-dimensional guidance at 4 different levels: level 1, annulus (parasternal short-axis view); level 2, sinuses of Valsalva; level 3, proximal AscAo 10 to $20 \mathrm{~mm}$ distal to the sinotubular junction (both parasternal long-axis views); and level 4, descending abdominal aorta just proximal to the branching off of the celiac trunk (abdominal paramedian longaxis view). Attention was paid to setting the line of sight exactly perpendicular to the long axis of the aorta in views showing the largest aortic diameters. Sharp endothelial lines were used as additional indicators for the line of sight to cut the central line of the aorta. Aortic dilatation was determined with standard nomograms. ${ }^{10}$

For automated and standardized calculation of aortic diameters, we developed suitable software. First, M-mode tracings of the AscAo (level 3) and DescAo (level 4) of at least 5 heart cycles were loaded into the program. To find the inner aortic wall contours, an image-processing algorithm ran on the M-mode images. Out of the determined aortic edge map, AscAo and DescAo outlines were calculated throughout the heart cycles (Figure 1, left). In some images with a suboptimal signal-to-noise ratio, minor manual corrections of aortic wall contours had to be performed. Interobserver reproducibility, calculated as the standard deviation of the differences between measurements and expressed as the percentage of the mean of the measurements, was determined after re-evaluation of randomly selected images by a second investigator blinded to the initial results. According to the usual aortic diameter measurements with the leading edge technique, ${ }^{10}$ the automatically detected inner diameter of the aorta was enlarged by the anterior aortic wall thickness. Time-diameter curves of 5 heart cycles were generated, based on the aortic wall contours. They showed a time resolution of approximately $6 \mathrm{~ms}$ per pixel and a spatial resolution of $0.2 \mathrm{~mm}$ per pixel. The curves were averaged and slightly smoothed by a digital low-pass filter (Butterworth; degree 2) to eliminate the digitalization noise (Figure 1, right). Out of time-diameter curves and averaged threefold blood pressure measurements, which were taken at the right arm oscillometrically (Dinamap; GE Healthcare, Slough, United Kingdom) immediately before M-mode registration, aortic elastic parameters were estimated automatically.

\section{Calculation of Aortic Parameters}

In addition to established aortic elastic parameters such as aortic distensibility and wall stiffness index, ${ }^{6,11}$ we developed maximum systolic area increase (MSAI), a parameter that is advantageous because of its easy determination. Aortic integral ratio and vectoraortography indicate the region of reduced aortic elasticity. The parameters were defined as follows.

Systolic diameter increase was calculated as 
TABLE 1. Aortic parameters and FBN1 gene mutations in 19 patients with Marfan syndrome

\begin{tabular}{|c|c|c|c|c|c|c|c|c|c|c|c|c|c|}
\hline \multirow{3}{*}{$\frac{\text { Patient No. }}{1^{*}}$} & \multirow{3}{*}{$\begin{array}{c}\text { Age } \\
\text { (y) }\end{array}$} & \multirow{3}{*}{$\frac{\text { Sex }}{F}$} & \multirow{3}{*}{$\frac{\text { BSA }\left(\mathrm{m}^{2}\right)}{0.59}$} & \multirow[b]{2}{*}{ Mutation } & \multirow{2}{*}{\multicolumn{2}{|c|}{$\begin{array}{c}\text { Aortic root } \\
\text { diastolic } \\
\text { diameter (mm, } \\
\text { dilated +) } \\
\end{array}$}} & \multirow{2}{*}{\multicolumn{2}{|c|}{$\begin{array}{c}\text { AscAo } \\
\text { diastolic } \\
\text { diameter (mm, } \\
\text { dilated +) }\end{array}$}} & \multirow{3}{*}{$\begin{array}{c}\begin{array}{c}\text { DescAo } \\
\text { diastolic } \\
\text { diameter } \\
(\mathbf{m m})\end{array} \\
5.2\end{array}$} & \multirow{3}{*}{$\begin{array}{c}\begin{array}{c}\text { Systolic } \\
\text { blood }\end{array} \\
\begin{array}{c}\text { pressure } \\
\text { (mm Hg) }\end{array} \\
90\end{array}$} & \multirow{3}{*}{$\begin{array}{c}\begin{array}{c}\text { Pulse } \\
\text { pressure } \\
\text { (mm Hg) }\end{array} \\
38\end{array}$} & \multicolumn{2}{|c|}{$\begin{array}{c}\text { Distensibility } \\
\left(\mathrm{kPa}^{-1} \cdot 10^{-3}\right)\end{array}$} \\
\hline & & & & & & & & & & & & AscAo & DescAo \\
\hline & & & & $344 C>G ; S 115 C \S$ & 20 & + & 14.4 & - & & & & 39 & 79 \\
\hline 2 & 2 & $\mathrm{~F}$ & 0.63 & $\begin{array}{l}\text { 1206delT; frameshift } \\
\text { + PTC }\end{array}$ & 21 & + & 15.4 & - & 7.7 & & 60 & 21 & 36 \\
\hline $3 \dagger$ & 9 & $\mathrm{~F}$ & 1.20 & $\begin{array}{l}\text { 508delT; frameshift }+ \\
\text { PTC }\end{array}$ & 27 & + & 18.5 & - & 12.1 & 111 & 58 & 70 & 56 \\
\hline $4 \dagger$ & 10 & $\mathrm{~F}$ & 1.21 & $\begin{array}{l}\text { 508delT; frameshift + } \\
\text { PTC }\end{array}$ & 28 & + & 20.1 & + & 12.3 & 96 & 57 & 24 & 40 \\
\hline $5 \ddagger$ & 11 & $\mathrm{~F}$ & 1.51 & 7801C > T; 02601X & 31 & + & 21.2 & - & 12.7 & 118 & 47 & 43 & 44 \\
\hline $6 \ddagger$ & 12 & $\mathrm{~F}$ & 1.78 & $7801 \mathrm{C}>\mathrm{T} ; 02601 \mathrm{X}$ & 37 & + & 22.8 & - & 11.5 & 122 & 46 & 61 & 72 \\
\hline 7 & 12 & $\mathrm{~F}$ & 1.40 & $1453 \mathrm{C}>\mathrm{T} ; \mathrm{R} 485 \mathrm{C}$ & 27 & - & 24.1 & + & 12.2 & 116 & 55 & 38 & 34 \\
\hline $8 \ddagger$ & 15 & $\mathrm{~F}$ & 1.95 & $7801 \mathrm{C}>\mathrm{T} ; 02601 \mathrm{X}$ & 33 & + & 28.2 & + & 11.8 & 116 & 55 & 22 & 48 \\
\hline $9 \dagger$ & 16 & $\mathrm{~F}$ & 1.63 & $\begin{array}{l}\text { 508delT; frameshift }+ \\
\text { PTC }\end{array}$ & 32 & + & 26.5 & + & 12.1 & 117 & 48 & 35 & 73 \\
\hline 10 & 16 & $\mathrm{M}$ & 1.81 & $\begin{array}{l}\text { No FBN1 mutation } \\
\text { detected }\end{array}$ & 34 & + & 23.1 & - & 10.9 & 116 & 54 & 21 & 76 \\
\hline 11 & 17 & $\mathrm{M}$ & 1.87 & IVS14-2A $>G \|$ & 36 & + & 25.8 & - & 17.4 & 117 & 61 & 20 & 35 \\
\hline 12 & 18 & $\mathrm{~F}$ & 1.87 & IVS45 + 3insCCI & 44 & + & 41.5 & + & 14.4 & 113 & 48 & 0 & 37 \\
\hline 13 & 18 & $\mathrm{M}$ & 1.87 & $\begin{array}{l}\text { 3194delAAAG; } \\
\text { frameshift + PTC }\end{array}$ & 42 & + & 31.9 & + & 15.0 & 137 & 62 & 0 & 48 \\
\hline 14 & 22 & $\mathrm{~F}$ & 1.63 & $\begin{array}{l}\text { 4337delATA; } \\
\text { D1446_I1447delinsV }\end{array}$ & 50 & + & 34.2 & + & 14.1 & 111 & 33 & 0 & 31 \\
\hline 15 & 23 & $\mathrm{~F}$ & 1.81 & $651 G>A ; W 217 X$ & 36 & + & 24.8 & - & 14.6 & 130 & 50 & 30 & 9 \\
\hline 16 & 25 & $\mathrm{~F}$ & 1.88 & $6794 G>A ; C 2265 Y$ & 36 & + & 27.3 & - & 17.3 & 120 & 64 & 67 & 14 \\
\hline 17 & 30 & $\mathrm{~F}$ & 1.76 & $2638 G>A ; G 880 S$ & 51 & + & 42.6 & + & 17.4 & 116 & 47 & 27 & 41 \\
\hline $18^{*}$ & 30 & $\mathrm{~F}$ & 1.85 & $344 C>G ; S 115 C \S$ & 32 & - & 28.7 & - & 12.7 & 129 & 46 & 0 & 35 \\
\hline $19 \ddagger$ & 40 & $\mathrm{~F}$ & 2.02 & $7801 \mathrm{C}>\mathrm{T} ; 02601 \mathrm{X}$ & 42 & + & 27.7 & - & 15.5 & 131 & 57 & 25 & 13 \\
\hline \multicolumn{14}{|l|}{ Patients } \\
\hline Mean & 17.7 & & 1.56 & & 34.7 & $\mathrm{n}=17$ & 26.2 & $\mathrm{n}=8$ & 13.0 & 116 & 52 & 29 & 43 \\
\hline SD & 9.5 & & 0.43 & & 8.5 & $89 \%$ & 7.5 & $42 \%$ & 3.1 & 11 & 8 & 21 & 21 \\
\hline \multicolumn{14}{|l|}{ Controls } \\
\hline Mean & 17.6 & & 1.44 & & 24.6 & $\mathrm{n}=0$ & 20.6 & $\mathrm{n}=1$ & 12.0 & 116 & 49 & 62 & 65 \\
\hline SD & 9.9 & & 0.39 & & 4.6 & & 4.0 & $5 \%$ & 2.6 & 8 & 9 & 24 & 30 \\
\hline$P$ value & NS & & NS & & $<.001$ & & .007 & & NS & NS & NS & $<0.001$ & 0.013 \\
\hline
\end{tabular}

BSA, Body surface area; AscAo, ascending aortic; DescAo, descending aortic; PTC, premature termination codon; SD, standard deviation; NS, not significant, + , Dilated; - , not dilated.

$*, \dagger$, and $\ddagger$ indicate the members of family 1,2 and 3 .

§Patients 1 and 18 carry a published mutation. ${ }^{29}$

|Cryptic splice site in intron 14 and insertion of $18 \mathrm{bp}$ in frame.

IDeletion of exon 45 in frame.

$$
\left(D_{\mathrm{s}}-D_{\mathrm{d}}\right) / D_{\mathrm{d}}(\%)
$$

where $D_{\mathrm{s}}$ is systolic (maximum) and $D_{\mathrm{d}}$ is end-diastolic (minimum) aortic diameter. Cross-sectional (CS) aortic distensibility and stiffness index were estimated as previously described ${ }^{6,11}$ :

$$
\begin{gathered}
\text { Distensibilty }=\frac{A_{\mathrm{s}}-A_{\mathrm{d}}}{A_{\mathrm{d}} \cdot\left(P_{\mathrm{s}}-P_{\mathrm{d}}\right) \cdot 1333} \cdot 10^{7}\left(\mathrm{kPa}^{-1} \cdot 10^{-3}\right) \\
\text { Stiffness index } \left.=\frac{\operatorname{In}\left(P_{\mathrm{s}} / P_{\mathrm{d}}\right)}{\left(D_{\mathrm{s}}-D_{\mathrm{d}}\right) / D_{\mathrm{d}}} \text { (dimensionless }\right),
\end{gathered}
$$

where $A_{\mathrm{s}}$ is systolic and $A_{\mathrm{d}}$ is end-diastolic area and $P_{\mathrm{s}}$ is systolic and $P_{\mathrm{d}}$ is diastolic blood pressure $(\mathrm{mm} \mathrm{Hg})$. Area $A$ was determined as $(D / 2)^{2} \cdot \pi$.

MSAI was defined as the maximum systolic slope of the area-time curve $A(\mathrm{t})$ normalized to $A_{\mathrm{d}}$ :

$$
\operatorname{MSAI}=\frac{d}{d t}\left[\left(A(\mathrm{t}) / A_{\mathrm{d}}-1\right) \cdot 100\right]_{\max }(\% / 100 \mathrm{~ms})
$$

Integrals of the AscAo and DescAo area-time curves normalized to the corresponding end-diastolic area-defined as aortic integral ratios-show in which aortic segment elasticity is reduced more severely. 
TABLE 2. Cardiovascular parameters in patients with Marfan syndrome (MFS) and in control subjects

\begin{tabular}{|c|c|c|c|}
\hline Parameters & $\begin{array}{c}\text { MFS } \\
(n=19)\end{array}$ & $\begin{array}{l}\text { Control subjects } \\
(\mathrm{n}=19)\end{array}$ & $P$ value \\
\hline Heart rate (beats/min) & $71 \pm 19$ & $73 \pm 14$ & NS \\
\hline Systolic blood pressure (mm Hg) & $116 \pm 11$ & $116 \pm 8$ & NS \\
\hline Diastolic blood pressure (mm Hg) & $65 \pm 12$ & $68 \pm 10$ & NS \\
\hline Pulse pressure $(\mathrm{mm} \mathrm{Hg})$ & $52 \pm 8$ & $49 \pm 9$ & NS \\
\hline \multicolumn{4}{|l|}{ Bulbus aortae } \\
\hline Diastolic diameter (mm) & $34.7 \pm 8.5$ & $24.6 \pm 4.6$ & $<.001$ \\
\hline Normalized diastolic diameter $\left(\mathrm{mm} / \mathrm{m}^{2}\right)$ & $22.9 \pm 5.1$ & $18.0 \pm 4.0$ & .002 \\
\hline \multicolumn{4}{|l|}{ Ascending aorta } \\
\hline Diastolic diameter (mm) & $26.2 \pm 7.5$ & $20.6 \pm 4.0$ & .007 \\
\hline Normalized diastolic diameter $\left(\mathrm{mm} / \mathrm{m}^{2}\right)$ & $17.0 \pm 4.1$ & $15.2 \pm 3.4$ & NS \\
\hline Systolic diameter increase (\%) & $7.5 \pm 10.6$ & $18.0 \pm 6.1$ & .001 \\
\hline Distensibility $\left(\mathrm{kPa}^{-1} \cdot 10^{-3}\right)$ & $29 \pm 21$ & $62 \pm 24$ & $<.001$ \\
\hline Stiffness index & $6.2 \pm 2.6$ & $3.4 \pm 1.4$ & $<.001$ \\
\hline Maximum systolic area increase (\%/100 ms) & $28 \pm 19$ & $55 \pm 21$ & $<.001$ \\
\hline \multicolumn{4}{|l|}{ Descending aorta } \\
\hline Diastolic diameter (mm) & $13.0 \pm 3.1$ & $12.0 \pm 2.6$ & NS \\
\hline Normalized diastolic diameter $\left(\mathrm{mm} / \mathrm{m}^{2}\right)$ & $8.4 \pm 1.6$ & $8.8 \pm 2.2$ & NS \\
\hline Systolic diameter increase (\%) & $13.6 \pm 5.9$ & $18.6 \pm 6.1$ & .014 \\
\hline Distensibility $\left(\mathrm{kPa}^{-1} \cdot 10^{-3}\right)$ & $43 \pm 21$ & $65 \pm 30$ & .013 \\
\hline Stiffness index & $5.8 \pm 3.9$ & $3.2 \pm 1.0$ & .009 \\
\hline Maximum systolic area increase (\%/100 ms) & $41 \pm 17$ & $54 \pm 21$ & .044 \\
\hline \multicolumn{4}{|l|}{ Vectoraortography } \\
\hline Magnitude (\%) & $18.2 \pm 6.2$ & $25.4 \pm 7.2$ & .002 \\
\hline Phase (degree) & $66 \pm 41$ & $47 \pm 11$ & NS \\
\hline Aortic integral ratio & $1.1 \pm 2.1$ & $1.1 \pm 0.5$ & NS \\
\hline
\end{tabular}

Values are shown as mean \pm SD; $N S$, Not significant $(P>.05)$; normalized diastolic diameter means diastolic diameter divided by body surface area.

$$
\text { Aortic integral ratio }=\frac{\int_{\mathrm{HC}}\left(A(\mathrm{t}) / A_{\mathrm{d}}\right)_{\mathrm{AscAo}} d t}{\int_{H C}\left(A(\mathrm{t}) / A_{\mathrm{d}}\right)_{\operatorname{DescAo}} d t},
$$

where $A(\mathrm{t})$ the is aortic area-time curve and $\mathrm{HC}$ is the heart cycle.

The vectoraortography visualizes the vector loop of the relative AscAo and DescAo diameter changes during the heart cycle. The rotating vector can be characterized by its magnitude and phase:

Magnitude(t)

$=\sqrt{\left[\left(D(\mathrm{t}) / D_{\mathrm{d}}-1\right) \cdot 100\right]_{\mathrm{AscAo}}^{2}+\left[\left(D(\mathrm{t}) / D_{\mathrm{d}}-1\right) \cdot 100\right]_{\text {DescAo }}^{2}}(\%)$,

$$
\text { Phase }(\mathrm{t})=\arctan \left(\frac{\left(D(\mathrm{t}) / D_{\mathrm{d}}-1\right)_{\text {DescAo }}}{\left(D(\mathrm{t}) / D_{\mathrm{d}}-1\right)_{\text {AscAo }}}\right)(\text { degree }),
$$

where $D(\mathrm{t})$ is the aortic diameter-time curve.

\section{Statistics}

Data are expressed as mean \pm SD and, in Figure 2, $A$ as mean $\pm 95 \%$ confidence interval. Quantitative variables were compared by means of unpaired Student $t$ tests and Mann-Whitney $U$ tests, respectively. The relation between continuous variables was tested by linear regression analysis. Single and multiple logistic regression models were developed to estimate the diagnostic power of the aortic parameters.
The conditional probability for the presence of MFS is denoted by the equation $P(\mathrm{MFS}=1)=1[1+\exp (-z)]$ where $z$ indicates the logit of the model. The effect of each model parameter is given by its odds ratio. All statistical analyses were performed with the software package SPSS 11.0 (SPSS Inc, Chicago, Ill).

\section{Results}

\section{Clinical Characteristics}

Clinical characteristics of the Marfan patients and control persons were compared in Tables 1 and 2. Age, sex, weight, height, body-surface area, and heart rate did not differ between Marfan patients and control persons. Four patients $(21 \%)$ presented with mild aortic regurgitation $(1+), 16$ $(84 \%)$ presented with mitral valve prolapse, and $16(84 \%)$ presented with mitral regurgitation ( 7 with $1+, 8$ with $2+$, and 1 with $3+$ ) as defined by Doppler echocardiography. Seventeen patients $(90 \%)$ showed skeletal symptoms of MFS; in $6(32 \%)$ of them the skeleton was involved, and 11 (58\%) fulfilled the major skeletal criteria according to the Ghent nosology. ${ }^{8}$ Ectopia lentis was present in 10 patients (53\%), and 11 patients (58\%) had a family history of MFS. Results of FBN1 gene mutation analysis are presented in Table 1 . 

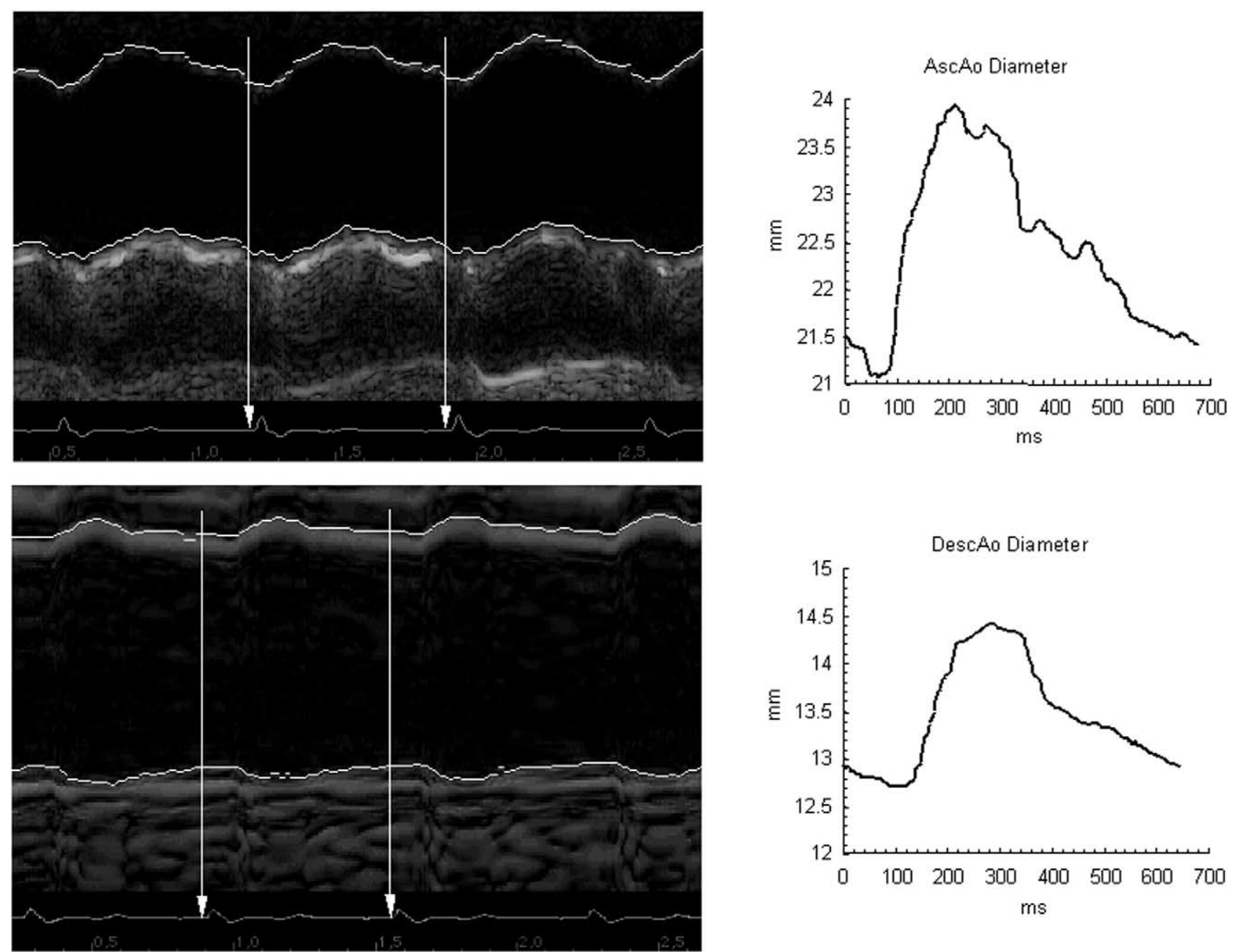

Figure 1. Semiautomated aortic wall contour analysis (white lines) in M-mode images of the ascending (top left) and descending (bottom left) aorta of patient 5. Arrows indicate the beginning and end of 1 heart cycle. Averaged time-diameter curves of the ascending (top right) and descending (bottom right) aorta are shown.

\section{Aortic Dimensions and Calculation of Elastic Parameters}

Echocardiographic aortic findings of the Marfan group and the control group are shown in Tables 1 and 2. Diastolic aortic root $(P<.001)$ and diastolic AscAo diameter $(P=$ $.007)$ were significantly increased in the MFS group, whereas the difference of DescAo diameters between groups did not reach statistical significance. All 4 investigated elastic parameters demonstrated reduced aortic elastic properties in MFS patients (Table 2 and Figure 2): AscAo systolic diameter increase ( $42 \%$ of control group), CS distensibility (47\%), and MSAI (51\%) were significantly diminished in the Marfan group. The stiffness index, as being inversely related to distensibility, was markedly increased (182\% of control group). Four MFS patients (patients 12-14 and 18; Table 1) revealed an AscAo diameter decrease during systole; in these cases, CS distensibility and MSAI were set to 0 , and stiffness index could not be calculated. Note that both patients without aortic root dilatation (patients 7 and 18; Table 1) showed a decreased AscAo distensibility and a reduced DescAo distensibility of $\geq 1 \mathrm{SD}$. In the DescAo of MFS patients, we observed less systolic diameter increase, CS distensibility, and MSAI; the stiffness index was markedly greater than in the control group. The differences were smaller than in the AscAo (Table 2). In 3 of 5 adult MFS patients, in whom elective prosthetic aortic root replacement was indicated at or 1 year after the initial investigation (patients 12, 14, and 17 out of the study group [Table 1] and 2 patients out of the validation group), AscAo distensibility and MSAI were 0 . Because of systolic diameter decrease, aortic stiffness index could not be calculated. In the remaining 2 of the 5 operated patients, AscAo dis- 

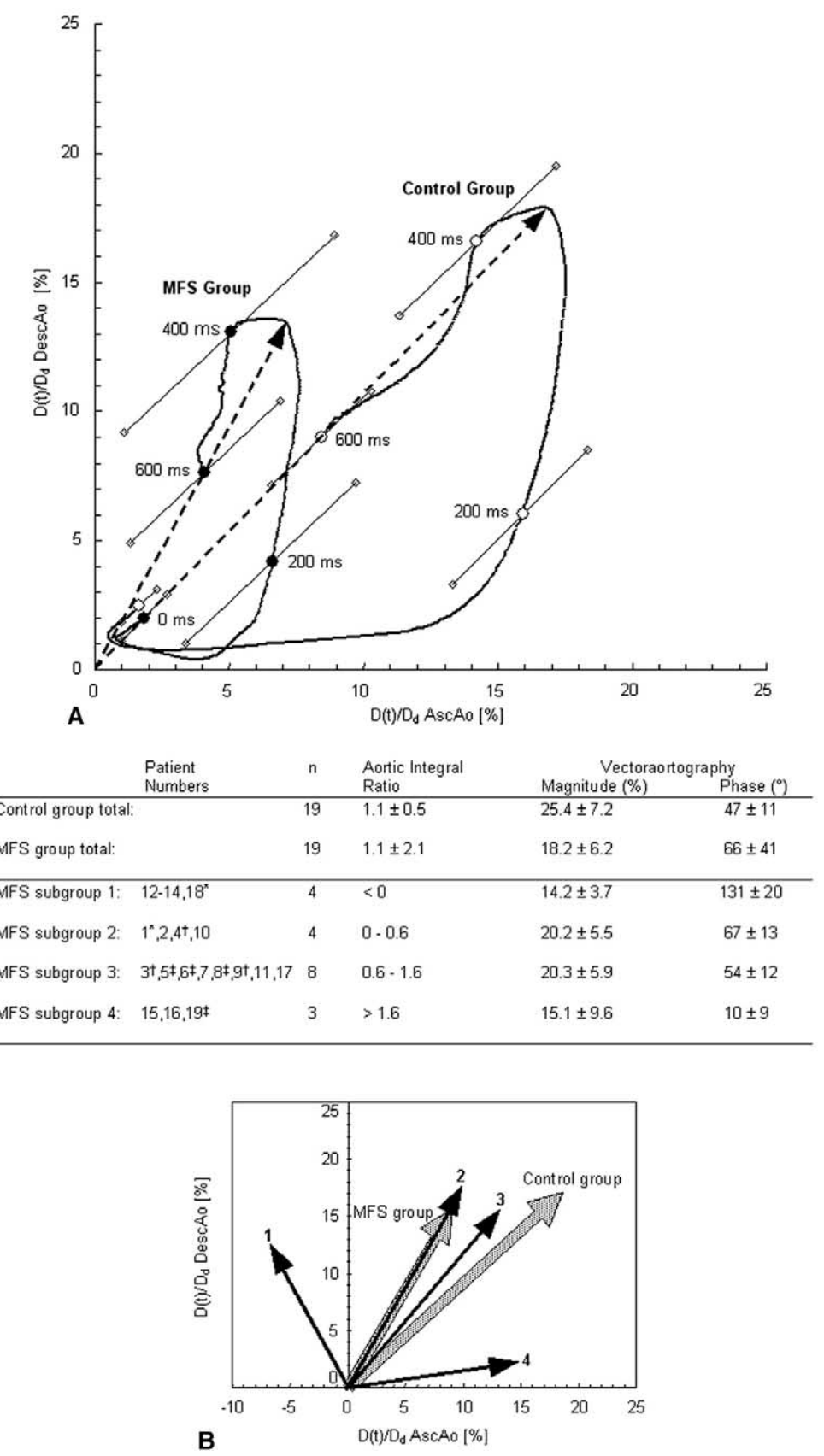

Figure 2. Vectoraortography. A, Vector loops represent relative ascending (AscAo) and descending (DescAo) aortic diameter changes $\left(D(t) / D_{\mathrm{d}}\right)$ during the heart cycle. The $95 \%$ confidence interval at $0,200,400$, and 600 ms is denoted by thin lines. The loop of the Marfan (MFS) patients is smaller and a little steeper than that of the control group. This shows the reduction of aortic elasticity predominantly present in the AscAo of MFS patients. Arrows indicate the vectors' maximum magnitude in the MFS and the control groups. Loops are not closed because of varying cycle lengths of individuals. Beyond 600 ms cycle length, data were not included. B, The MFS group was divided into 4 subgroups according to the aortic integral ratio. The vectors of the total MFS group, the MFS subgroups, and the control group are shown. * $t$, and $\neq$ indicate the members of family 1, 2, and 3 (Table 1).

tensibility was strongly decreased $\left(12\right.$ and $\left.27 \mathrm{kPa}^{-1} \cdot 10^{-3}\right)$. However, 1 MFS patient (patient 18) showed an AscAo 0 distensibility without aortic root dilatation.
AscAo and DescAo CS distensibility were greater in young patients and control persons (age, 2-12 years; $\mathrm{n}=7$ ) than in older ones (age, 15-19 years; $n=6$; and age, 20-42 
years; $\mathrm{n}=6$ ), although no strong linear correlation between age and distensibility could be found $(r<0.8)$. In MFS patients, the differences between age groups were less pronounced than in controls.

CS distensibility and MSAI values of the AscAo and DescAo of Marfan patients and control persons correlated significantly $(\mathrm{MSAI}=0.68 \times \mathrm{CS}$ distensibility $+10.54 ; r=0.86$; $P<.01)$.

Interobserver reproducibility, which was determined in 6 consecutive patients, was $2.6 \%$ and $3.3 \%$ for AscAo and DescAo diastolic diameter measurements and was $3.8 \%$ and 4.6\% for AscAo and DescAo distensibility. Reproducibility of further aortic elastic parameters showed comparable values.

\section{Aortic Integral Ratio}

Mean values of the AscAo/DescAo integral ratio were similar between Marfan patients and control persons, but in the MFS group, the standard deviation was markedly increased $(1.1 \pm 2.1$ in the MFS vs $1.1 \pm 0.5$ in the control group; Table 2). This ratio showed the variable extent of regional aortic elasticity alterations in the Marfan patients and, conversely, a tight relationship of AscAo and DescAo integrals in healthy control subjects.

\section{Vectoraortography}

The vector loops characterizing the relative aortic diameter changes during the heart cycle differed significantly between the MFS and the control group (Figure 2, $A$ and Table 2 ). The maximum magnitude of the vector (Figure 2, $A$ ) was significantly reduced in the MFS group, and the vector's phase at maximum magnitude (ie, the angle below the vector) showed no significant difference between groups $(P$ $=.061)$. Because of the high standard deviation of the phase and aortic integral ratio in the MFS group, we split the Marfan patients into 4 subgroups to distinguish among different elasticity patterns (Figure 2, B). In the first subgroup, the AscAo diameter decreased during early systole, so that phase was strongly increased (mean, $131^{\circ}$ ). In subgroup 2, phase was also increased, but AscAo diameter increased during systole. Subgroup 3 (aortic integral ratio, 0.6-1.6; ie, mean value $\pm 1 \mathrm{SD}$ of control group) showed a mean phase $\left(54^{\circ}\right)$ roughly comparable to the control group because of similar reduction of AscAo and DescAo pulsatile diameter changes. In subgroup 4, phase was strongly reduced (mean $10^{\circ}$ ) because of decreased pulsatile diameter changes predominantly in the DescAo.

\section{Single and Multiple Logistic Regression Analysis}

All presented aortic parameters were tested separately for their diagnostic power by single logistic regression analysis (Table 3). AscAo distensibility and systolic diameter increase demonstrated the highest sensitivity (84\%); the dia- stolic diameter of the bulbus aortae normalized to bodysurface area showed the highest specificity $(84 \%)$.

To increase the diagnostic power of the classification models, we searched for the multiple logistic regression model $z$ displaying the highest sensitivity $(94.7 \%)$ and specificity $(100 \%)$. The logit of the regression model is given by the following equation:

$[z=4.379+2.293 \cdot$ normalized BA diastolic diameter

- 2.449 . normalized AscAo diastolic diameter

$-0.247 \cdot$ AscAo distensibility]

$(P=.030 ; P=.028 ; P=.035$; odds ratios: 9.901, 0.086, and 0.781; Table 3)

Subsequently, our best model $z$ was tested on the independent validation group and showed a sensitivity of $100 \%$ and a specificity of $94.7 \%$. Validation of the single logistic regression models also yielded comparable results to those established in the study population.

\section{Discussion}

Our new noninvasive semiautomated M-mode echocardiographic image-segmentation technique showed reduced aortic elastic properties in children and young adults with MFS, with high accuracy and objectivity. In most published studies, aortic diameters and CS areas, which underlie the calculation of aortic elastic properties, still rely on a slow, tedious, and observer-dependent process of manual outlining, which has to be performed by expert physicians.

In adults with MFS, automated border detection has been used to measure aortic diameters out of transesophageal aortic images. ${ }^{12}$ We used 2-dimensional guided transthoracic M-mode echocardiographic aortic diameter measurements, which showed good correlation with 2-dimensional echocardiographically obtained values. ${ }^{10}$ Two-dimensional guidance is indispensable for correct diameter measurements out of M-mode echocardiographic images, especially for displaying the largest aortic diameter and for finding an axis strictly perpendicular to the long axis of the aorta. ${ }^{10}$ In contrast to continuous aortic measurements out of 2dimensional echocardiographic or magnetic resonance imaging sequences, M-mode echocardiography enables us to measure aortic diameters over 5 heart cycles with twofold to fivefold higher time resolution out of merely 1 to 2 images. In children and young adults, images of high quality can be obtained in most cases. However, accurate image acquisition with a high signal-to-noise ratio is essential for appropriate computerized contour finding.

Aortic root dilatation, a major criterion of MFS, ${ }^{8}$ was shown to be present in $89 \%$ of our patients and was reported in $61.5 \%$ to $84 \%$ of adults ${ }^{1,13-16}$ and in $42.5 \%$ to $76 \%$ of children aged 0.25 to 18 years. ${ }^{15,17}$ AscAo dilatation was present in $42 \%$ of our patients and has been reported in $54 \%$ of adults ${ }^{15}$ and in $45 \%$ of children (age, $0.5-18$ years). ${ }^{15}$ 
TABLE 3. Predictive power of single and multiple logistic regression models

\begin{tabular}{|c|c|c|c|}
\hline Cardiovascular parameters & Sensitivity (\%) & Specificity (\%) & $\begin{array}{c}\boldsymbol{P} \text { value of } \\
\text { parameter(s) }\end{array}$ \\
\hline \multicolumn{4}{|l|}{ Single logistic models } \\
\hline \multicolumn{4}{|l|}{ Bulbus aortae (BA) } \\
\hline Normalized diastolic diameter $\left(\mathrm{mm} / \mathrm{m}^{2}\right)$ & 63.2 & 84.2 & .013 \\
\hline \multicolumn{4}{|l|}{ Ascending aorta (AscAo) } \\
\hline Normalized diastolic diameter $\left(\mathrm{mm} / \mathrm{m}^{2}\right)$ & 47.4 & 68.4 & NS \\
\hline Systolic diameter increase (\%) & 84.2 & 68.4 & .006 \\
\hline Distensibility $\left(\mathrm{kPa}^{-1} \cdot 10^{-3}\right)$ & 84.2 & 68.4 & .002 \\
\hline Stiffness index & 73.3 & 78.8 & .006 \\
\hline Maximum systolic area increase (MSAI) (\%/100 ms) & 68.4 & 68.4 & .003 \\
\hline \multicolumn{4}{|l|}{ Descending aorta (DescAo) } \\
\hline Normalized diastolic diameter $\left(\mathrm{mm} / \mathrm{m}^{2}\right)$ & 52.6 & 47.4 & NS \\
\hline Systolic diameter increase (\%) & 63.2 & 68.4 & .027 \\
\hline Distensibility $\left(\mathrm{kPa}^{-1} \cdot 10^{-3}\right)$ & 73.7 & 57.9 & .022 \\
\hline Stiffness index & 68.4 & 73.7 & .021 \\
\hline Maximum systolic area increase (MSAI) (\%/100 ms) & 52.6 & 57.9 & NS \\
\hline \multicolumn{4}{|l|}{ Vectoraortography } \\
\hline Magnitude (\%) & 73.7 & 63.2 & .007 \\
\hline Phase (degree) & 63.2 & 68.4 & NS \\
\hline Aortic integral ratio & 73.7 & 47.4 & NS \\
\hline \multicolumn{4}{|l|}{ Multiple logistic model (best model) } \\
\hline $\begin{array}{l}\text { Normalized BA diastolic diameter; normalized AscAo diastolic diameter; } \\
\text { AscAo distensibility }\end{array}$ & 94.7 & 100 & $.030 ; .028 ; .035$ \\
\hline
\end{tabular}

The diagnostic performance of the models is indicated by their sensitivity and specificity. The $P$ value describes the significance of each model's parameter(s). AscAo and DesAo diastolic diameters were normalized to body surface area $\left(\mathrm{mm} / \mathrm{m}^{2}\right)$.

Because aortic dilatation evolves during childhood and adolescence, serial evaluations of aortic dimensions may be necessary to clearly demonstrate the presence and progression of aortic dilatation. ${ }^{18}$ Because aortic root growth is of prognostic value for the occurrence of aortic complications, ${ }^{18}$ objective diameter measurements will enhance the accuracy of results.

The representation of time-diameter curves gives us an optical impression of aortic diameter changes during the heart cycle (Figure 1, right). The vectoraortography-a compaction of time-diameter relations of 2 aortic segments in 1 diagram-and the aortic integral ratio allow us to distinguish different patterns of aortic stiffening within the MFS group (Figure 2). In 4 patients with considerable aortic root dilatation, the AscAo anteroposterior diameter decreased during systole, which-to our knowledge- has never been described before (patients 12-14 and 18; mean end-diastolic aortic root diameter, $42.0 \pm 7.5 \mathrm{~mm}$ vs $34.9 \pm$ $8.6 \mathrm{~mm}$ in the total MFS group; mean end-diastolic AscAo diameter, $34.0 \pm 5.4 \mathrm{~mm}$ vs $26.2 \pm 7.5 \mathrm{~mm}$ in the total MFS group; Figure 2, $B$, subgroup 1). As we observed by echocardiography in a few patients with excellent quality of AscAo 2-dimensional images, the AscAo seemed to bump against an anterior structure (probably the sternum) during its systolic anterior movement; the aortic CS area for a short time deviated from its circular shape toward an elliptic shape. Therefore, the aortic wall of these patients is exposed to increased shear stress. AscAo distensibility and MSAI were set to 0 , and AscAo stiffness index could not be calculated. Patients with predominant loss of DescAo elasticity (subgroup 4) may resemble those who are at risk for aneurysm or dissection of the DescAo. ${ }^{19,20}$ Our technique can thus serve as a valuable noninvasive tool for assessing the descending abdominal aorta.

Simultaneous diameter and blood pressure registration is essential for exact calculation of elastic parameters. Simultaneous diameter and pressure registration at the same aortic site is impossible if elastic parameters are determined noninvasively. However, close correlation of invasive and noninvasive determination of AscAo distensibility has been demonstrated. ${ }^{5}$ Nevertheless, aortic valve competence and normal left ventricular systolic function are basic requirements for the interpretation of calculated aortic elastic parameters.

Several authors have shown decreased aortic distensibility and increased aortic stiffness index in patients with MFS. ${ }^{6,7,12,21-25}$ Data obtained in children are rare. ${ }^{6,21}$ Our results, which show a 50\% reduced AscAo and a 30\% reduced DescAo distensibility in the MFS group, compare well to published data on children ${ }^{6}$ and young adults ${ }^{7,22}$ 
with MFS. Smaller values of mean aortic distensibility were reported in older patients, ${ }^{24-27}$ and greater values were reported in younger children. ${ }^{21}$ Our data confirm this dependence of aortic distensibility on age. It is interesting to note that the patients with normal diameters of the bulbus and the AscAo also showed aortic dysfunction in terms of decreased AscAo and DescAo distensibility. Therefore, assessment of aortic dysfunction is of additional diagnostic value compared with AscAo diameter measurements. The necessity of $\beta$-blocker therapy should be discussed in those patients. MSAI is a further elastic parameter that is easy to determine, because blood pressure measurement is not required. In our series, MSAI correlated very closely with aortic distensibility. Follow-up investigations with the presented elastic parameters could prove the efficiency of medical treatment with, eg, $\beta$-blocking agents and may be of help in the timing of elective aortic surgery, especially in children and adolescents not presenting with excessively dilated aortic diameters that are unquestionably an indication for elective prosthetic aortic root replacement. In our opinion, an AscAo 0 distensibility can be regarded as additional argument for elective aortic surgery. More detailed clinical description was thought to be necessary to allow a genotype/phenotype correlation between patients described by other groups ${ }^{28}$; our results in this relatively small MFS group, however, did not reveal a dependence of aortic distensibility on the type of FBN1 mutation (data not shown). Objective data on aortic elastic properties, together with the results of FBN1 gene mutation analysis of a greater patient population, will perhaps show certain relationships. Because FBN1 mutation analysis is still too expensive and time-consuming to be used as screening tool, our logistic regression models based on the results of only aortic parameters are an alternative approach to recognize and classify MFS. In patients with suspected MFS without aortic dilatation, they can serve as useful additional diagnostic tools to decide whether these patients should be genetically tested. Our best multiple logistic regression model showed higher sensitivity $(94.7 \%)$ and specificity $(100 \%)$ than the best single logistic regression models (sensitivity and specificity of $68 \%-84 \%$ ). This validated multiple logistic regression model can predict MFS more reliably than a cardiologic investigation including only aortic diameter measurements (yielding a sensitivity of $89 \%$ in our population and $61 \%-84 \%$ in published patient populations). ${ }^{1,13-16}$ It helps to decide about the necessity of time-consuming follow-up investigations, especially in patients with low suspicion of MFS and normal aortic elasticity, but does not replace ophthalmologic and orthopedic investigations, because some rare patients with MFS show no aortic involvement. ${ }^{14,17}$ Patients with Ehlers-Danlos syndrome type IV $^{30}$ and thoracic aortic aneurysm ${ }^{2}$ may show reduced aortic elastic properties, too, and therefore may be investigated with similar logistic regression models.

\section{Conclusions}

In summary, we determined decreased aortic elastic properties in young patients with MFS by a standardized semiautomated image-segmentation technique that enables us to estimate AscAo and DescAo distensibility, stiffness index, and MSAI with high reproducibility. It also gives way to high-quality follow-up investigations of aortic elastic properties in patients with suspected or confirmed MFS. Vectoraortography illustrates and the aortic integral ratio quantifies the relationship of AscAo and DescAo elasticity and so may show the region at risk for severe aortic complications. Our multiple logistic regression model enables us to calculate the probability for the presence of MFS on the basis of the results of solely aortic parameters (distensibility, normalized diastolic diameters of aortic bulbus, and AscAo) and so can be used as a diagnostic tool with high predictive power. Follow-up investigations in a larger patient population will prove the efficiency of medical treatment and may determine the value of this method for the prediction of aortic dissection and rupture, so these elastic parameters may serve as additional criteria to indicate elective surgical intervention.

We thank Dr Peter Oefner (Stanford University) for the initial mutational analysis of patient 13 by denaturing high-performance liquid chromatography, Dr Barbara Utermann (Innsbruck Medical University) for genetic counseling of several patients, Melanie Maudrich for laboratory assistance, and Karin Kirchner and Silvia Achenrainer for secretarial assistance.

\section{References}

1. Pyeritz RE, McKusick VA. The Marfan syndrome: diagnosis and management. N Engl J Med. 1979;300:772-7.

2. Pyeritz RE, Dietz HC. Marfan syndrome and other microfibrillar disorders. In: Royce PM, Steinmann B, editors. Connective tissue and its heritable disorders: molecular, genetic and medical aspects. 2nd ed. New York: Wiley-Liss; 2002. p. 585-626.

3. Murdoch JL, Walker BA, Halpern BL, Kuzma JW, McKusick VA. Life expectancy and causes of death in the Marfan syndrome. $N$ Engl J Med. 1972;286:804-8.

4. Yin FCP, Brin KP, Ting CT, Pyeritz RE. Arterial hemodynamic indexes in Marfan's syndrome. Circulation. 1989;79:854-62.

5. Stefanadis C, Stratos C, Boudoulas H, Kourouklis C, Toutouzas P. Distensibility of the ascending aorta: comparison of invasive and non-invasive techniques in healthy men and in men with coronary artery disease. Eur Heart J. 1990;11:990-6.

6. Savolainen A, Keto P, Hekali P, Nisula L, Kaitila I, Vitasalo M, et al. Aortic distensibility in children with the Marfan syndrome. Am J Cardiol. 1992;70:691-3.

7. Hirata K, Triposkiadis F, Sparks E, Bowen J, Wooley CF, Boudoulas H. The Marfan syndrome: abnormal aortic elastic properties. J Am Coll Cardiol. 1991;18:57-63.

8. De Paepe A, Devereux RB, Dietz HC, Hennekam RCM, Pyeritz RE. Revised diagnostic criteria for the Marfan syndrome. Am J Med Genet. 1996;62:417-26.

9. Mátyás G, De Paepe A, Halliday D, Boileau C, Pals G, Steinmann B. Evaluation and application of denaturing HPLC for mutation 
detection in Marfan syndrome: identification of 20 novel mutations and two novel polymorphisms in the FBN1 gene. Hum Mutat. 2002;19:443-56.

10. Roman MJ, Devereux RB, Kramer-Fox R, O'Loughlin J. Two-dimensional echocardiographic aortic root dimensions in normal children and adults. Am J Cardiol. 1989;64:507-12.

11. Kawasaki T, Sasayama S, Yagi SI, Asakawa T, Hirai T. Non-invasive assessment of the age related changes in stiffness of major branches of the human arteries. Cardiovasc Res. 1987;21:678-87.

12. Franke A, Mühler EG, Klues HG, Peters K, Lepper W, von Bernuth G, et al. Detection of abnormal aortic elastic properties in asymptomatic patients with Marfan syndrome by combined transoesophageal echocardiography and acoustic quantification. Heart. 1996;75:307-11.

13. Peters KF, Kong F, Horne R, Francomano CA, Biesecker BB. Living with Marfan syndrome I. Perceptions of the condition. Clin Genet. 2001;60:273-82

14. Bruno L, Tredici S, Mangiavacchi M, Colombo V, Mazzotta GF, Sirtori CR. Cardiac, skeletal, and ocular abnormalities in patients with Marfan's syndrome and in their relatives: comparison with the cardiac abnormalities in patients with kyphoscoliosis. Br Heart J. 1984;51: 220-30.

15. Roman MJ, Rosen SE, Kramer-Fox R, Devereux RB. Prognostic significance of the pattern of aortic root dilation in the Marfan syndrome. J Am Coll Cardiol. 1993;22:1470-6.

16. Loeys B, Nuytinck L, Delvaux I, De Bie S, De Paepe A. Genotype and phenotype analysis of 171 patients referred for molecular study of the fibrillin-1 gene FBN1 because of suspected Marfan syndrome. Arch Intern Med. 2001;161:2447-54.

17. Lipscomb KJ, Clayton-Smith J, Harris R. Evolving phenotype of Marfan's syndrome. Arch Dis Child. 1997;76:41-6.

18. Groenink M, Rozendaal L, Naeff MSJ, Hennekam RCM, Hart AAM, van der Wall EE, et al. Marfan syndrome in children and adolescents: predictive and prognostic value of aortic root growth for screening for aortic complications. Heart. 1998;80:163-9.

19. Finkbohner R, Johnston D, Crawford S, Coselli J, Milewicz DM. Marfan syndrome: long-term survival and complications after aortic aneurysm repair. Circulation. 1995;91:728-33.
20. Gott VL, Cameron DE, Alejo DE, Greene PS, Shake JG, Caparrelli DJ, et al. Aortic root replacement in 271 Marfan patients: a 24-year experience. Ann Thorac Surg. 2002;73:438-43.

21. Reed CM, Fox ME, Alpert BS. Aortic biomechanical properties in pediatric patients with the Marfan syndrome, and the effects of atenolol. Am J Cardiol. 1993;71:606-8.

22. Jeremy RW, Huang H, Hwa J, McCarron H, Hughes CF, Richards JG. Relation between age, arterial distensibility, and aortic dilatation in the Marfan syndrome. Am J Cardiol. 1994;74:369-73.

23. Adams JN, Brooks M, Redpath TW, Smith FW, Dean J, Gray J, et al. Aortic distensibility and stiffness index measured by magnetic resonance imaging in patients with Marfan's syndrome. Br Heart J. 1995; 73:265-9.

24. Haouzi A, Berglund H, Pelikan PCD, Maurer G, Siegel RJ. Heterogeneous aortic response to acute $\beta$-adrenergic blockade in Marfan syndrome. Am Heart J. 1997;133:60-3.

25. Rios AS, Silber EN, Bavishi N, Varga P, Burton BK, Clark WA, et al. Effect of long-term $\beta$-blockade on aortic root compliance in patients with Marfan syndrome. Am Heart J. 1999;137:1057-61.

26. Groenink M, de Roos A, Mulder BJM, Spaan JAE, van der Wall EE. Changes in aortic distensibility and pulse wave velocity assessed with magnetic resonance imaging following beta-blocker therapy in the Marfan syndrome. Am J Cardiol. 1998;82:203-8.

27. Jondeau G, Boutouyrie P, Lacolley P, Laloux B, Dubourg O, Bourdarias JP, et al. Central pulse pressure is a major determinant of ascending aorta dilation in Marfan syndrome. Circulation. 1999;99: 2677-81.

28. Pepe G, Giusti B, Evangelisti L, Porciani MC, Brunelli T, Giurlani L, et al. Fibrillin-1 (FBN1) gene frameshift mutations in Marfan patients: genotype-phenotype correlation. Clin Genet. 2001;59:444-50.

29. Katzke S, Booms P, Tiecke F, Palz M, Pletschacher A, Türkmen S, et al. TGGE screening of the entire FBN1 coding sequence in 126 individuals with Marfan syndrome and related fibrillinopathies. Hum Mutat. 2002;20:197-208.

30. Steinmann B, Royce PM, Superti-Furga A. The Ehlers-Danlos syndrome. In: Royce PM, Steinmann B, editors. Connective tissue and its heritable disorders: molecular, genetic and medical aspects. 2nd ed. New York: Wiley-Liss; 2002. p. 431-523. 